\title{
New Scaling Limitation of the Floating Gate Cell in NAND Flash Memory
}

\author{
Yong Seok Kim, Dong Jun Lee, Chi Kyoung Lee, Hyun Ki Choi, Seong Soo Kim, Jai Hyuk Song, \\ Du Heon Song, Jeong-Hyuk Choi, Kang-Deog Suh* and Chilhee Chung. \\ NAND Flash Process Architecture Team, Semiconductor Business Division, Samsung Electronics Co., \\ San \#24, Nongseo-Dong, Giheung-Gu, Yongin-City, Gyunggi-Do 446-711, Korea \\ *School of Information and Communication Engineering, Sungkyunkwan University, Suwon, 440-746, South Korea.
} Tel) 82-31-209-3546, Email) yongseok7.kim@samsung.com

\begin{abstract}
As the scaling in NAND Flash Memory is progressed, the various interferences among the adjacent cells are more and more increased and the new phenomenon which is ignored until now has to be considered. In this paper, we will introduce the new program interference phenomenon which is generated between the program word line and the adjacent word lines along the bit-line. This new program interference is that the Vth's of the adjacent word lines along the bit-line are decreased while a word line is programming. Because this phenomenon is severely aggravated as the gate space is decreased, we have to consider this program interference for the future technology nodes.
\end{abstract}

Keywords- Scaling Limitation, interference, Floating Gate Type NAND Flash

\section{INTRODUCTION}

In many papers, the scaling limitations of the floating gate NAND flash cell are listed and most of all are related with the increment of the interference among the neighboring cells and the decrement of coupling ratio [1]. Especially, the cell to cell interference, which has been attributed to the parasitic capacitance coupling effect, is a biggest barrier of MLC operations [2]. This cell-to-cell coupling between the floating gates has been studied analytically by involving the size of the cell transistor and the operation bias conditions [3] and recently, the new concept of the direct field effect interference, which is generated between the adjacent channels of cell transistors, is introduced [4]. Despite of these scaling problems, the scaling of floating gate type NAND flash has been continued to sub$30 \mathrm{~nm}$ through Error Code Correction and program algorithm progress [5], [6]. In this paper, we will introduce the new program interference, which has never been mentioned in the published paper so far. This program interference is the Vth reduction of the adjacent high state cells while the adjacent cells are programming. In the way that this program interference is due to the charge loss by the leakage current, it is different from the well-known interference by the cell-to-cell coupling effect, which has to do with the potential induced by the charge in neighbor floating gate. But because this new phenomenon is generated between the program word line and the adjacent word lines along the bit-lines, we call this phenomenon the program interference. This program interference is very much increased as the space between the adjacent cells along the bit-line is decreased and is more serious in the fresh cells than in the cycled cells.

\section{EXPERIMENT RESULTS}

As shown in Fig.1, when WL(n) is programmed to the high state, the high program voltage is applied to the control gate of WL(n) and the pass voltage is to other control gates for inhibiting the programming of the unselected strings and for turning on the cells of the selected string [7]. Also, conventionally, NAND flash device is programmed from lower word line which is closer to the $\mathrm{CSL}$ (Common Sense Line) to higher word line which is closer to the BL(Bit Line). Although we had observed this program interference phenomenon in the main chip under the low pass voltage for the first time, we had conducted experiments with the test modules located beside the main cell, which is $35 \mathrm{~nm}$ technology node. The test module is easy to control and trace each cell. Also the test modules with different design rule can be tested under the same condition. The test modules of n-type floating gate cell have 4 bit-lines and 64 word-lines as shown in Fig. 1. The control signals of the test modules are generated from external signal generators and measured by HP4083.

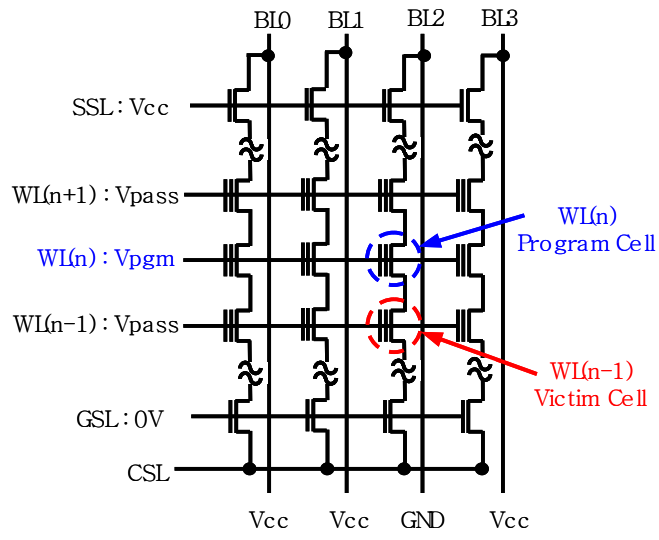

Figure 1. The schematic diagram for the test module with 4 bit-line and the basic program condtion for the local boosting scheme. 
At first, we erased all word lines in the test module and programmed only the WL(n-1) of BL2, which the target Vth of the erased cell is about $-3 \mathrm{~V}$ and the $\mathrm{Vth}$ of the programmed cell is about 4.7V. Next, we applied Vpgm to the WL(n) and Vpass to all other word lines and then we monitored the Vth with the stress time. The bias condition of this program stress is same as the condition for the normal program except for the amplitude of the voltage and the time. The Fig. 2 is a measured result for a single cell. At the first stage of the program stress, the program stress cell, WL(n), is being programmed and the Vth of the victim cell, WL(n-1), is being increased due to the cellto-cell coupling between the program stress cell and the victim cell. Next, the Vth of the program stress cell is saturated, which is due to the leakage of the inter-poly dielectric layer, and the Vth of victim cell is deceased. Although this program interference don't distinguish between the WL(n+1) and WL(n$1)$, we call the $\mathrm{WL}(\mathrm{n}-1)$ the victim cell because the program is done from the low word line to the high word line. In this paper, we will focus on the victim cell's behavior. The Fig. 3 is the measured results for 40 cells, where the Vth reductions have a wide distribution. In fact, at the main cell, this program interference phenomenon are observed as the under tail of the Vth distribution when all word line are programmed to high state in the multi-level cell operation. Because we are unfamiliar with this new phenomenon, we have confirmed whether it is dependent upon the back pattern, which means the Vth of other word lines except the program stress cell and the victim cell, and the sequence of program. But this phenomenon is only related to the adjacent cells along the bit-line. We have confirmed that this phenomenon doesn't distinguish between the upper word line, WL(n+1), and the under word line, WL(n1). To understand this phenomenon, we have investigated the dependence of the program stress voltage, the pass voltage, and the initial Vth of the victim cell. As shown in Fig. 4, we have measured the $\mathrm{Vth}$ of victim cell with the various program stress voltage. To magnify the difference of voltage between the neighboring gates, we have applied the low pass voltage, which is $4.5 \mathrm{~V}$. The decrement of the victim cell's Vth is getting larger with the program stress voltage increase. We have measured the same cells for the program stress voltage $24 \mathrm{~V}, 26 \mathrm{~V}$, and $28 \mathrm{~V}$ after initializing the cell and the each Vth in Fig. 4 is the average values for 20 cells.

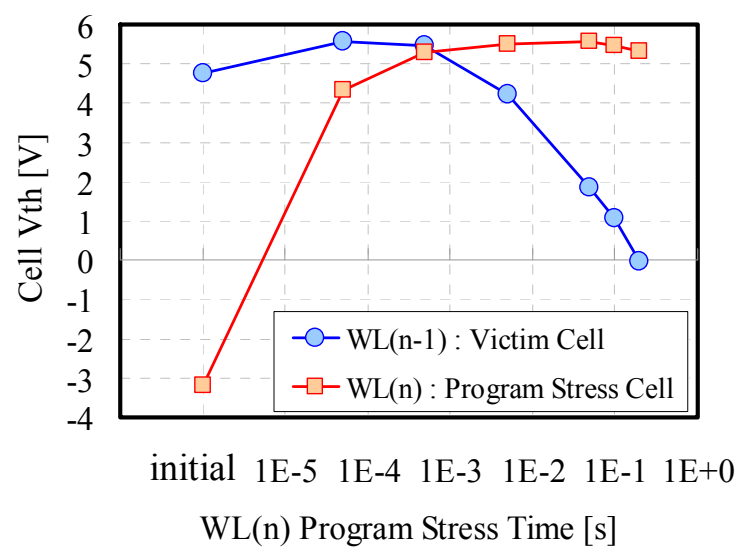

Figure 2. The Vth's of WL(n-1) and WL(n) with the program stress time for cells. The program stress voltage is $28 \mathrm{~V}$ and the pass voltage is $4.5 \mathrm{~V}$.

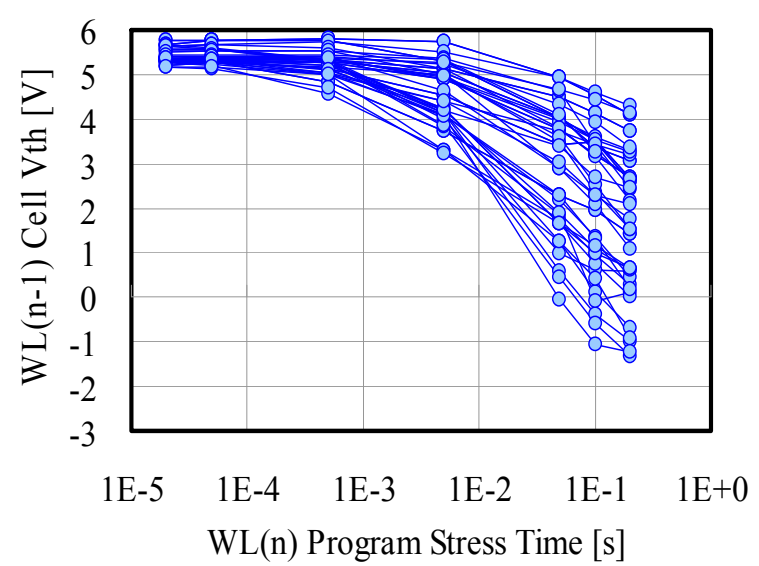

Figure 3. The Vth reduction of the victim cells, WL(n-1), with the program stress times for 40 cells. The program stress voltage is $26 \mathrm{~V}$ and the pass voltage is $4.5 \mathrm{~V}$.

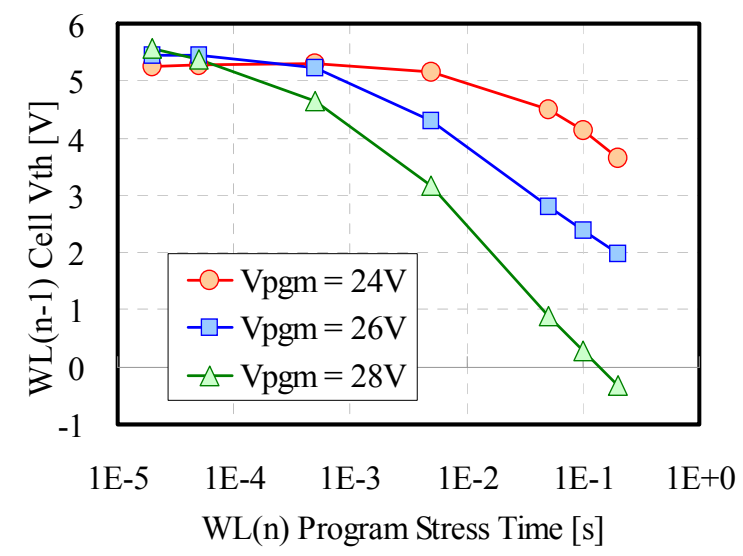

Figure 4. The Vth reduction of the victim cell, WL(n-1), with the program voltages, which are 24,26 , and $28 \mathrm{~V}$. The pass voltage is $4.5 \mathrm{~V}$.

The Fig. 5 is the measured results of the victim cell's Vth with the various Vpass. We have measured at the same cells for the pass voltage $4.5 \mathrm{~V}, 7 \mathrm{~V}$, and $10 \mathrm{~V}$ after initializing the cell repeatedly, and the results of each $\mathrm{WL}(\mathrm{n}-1) \mathrm{Vth}$ in Fig. 5 are the average of 20 cells. Contrary to the program voltage experiment, the decrement of the victim cell's Vth is getting larger as the pass voltage decreases. This is the reason why the low pass voltage is used when the experiment for this program interference is performed. The potential difference between the control gate and the floating gate can be changed by altering the initial cell Vth of the victim cell. The charge loss of the victim cell decreases as the initial cell Vth of the victim cell decreases. The results in Fig. 6 are measured at the same cell after the Vth of the cells is adjusted again. Although the initial Vth's of the victim cell are different, it appears as if the victim cell's Vth converges as program stress time increases, as shown in Fig. 6. Some cells exactly converge into a single values and other only gathered at a range. This convergence is well observed at high program stress voltage and in cells with small spaces between the gates. 


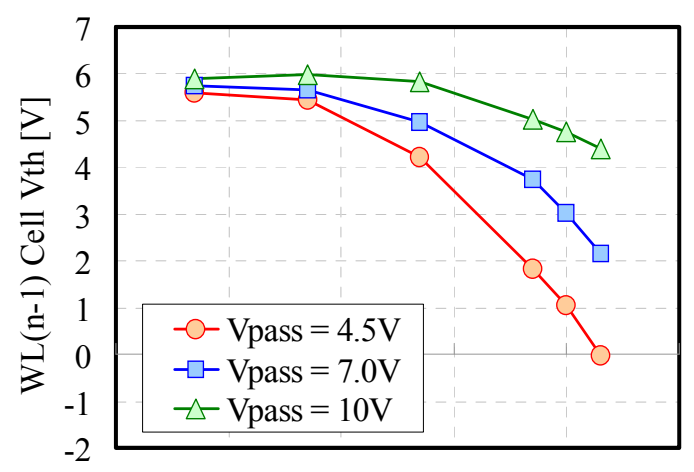

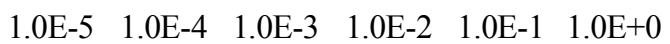
WL(n) Program Stress Time [s]

Figure 5. The Vth's reduction of WL(n-1) with the Vpass. The program voltage is $28 \mathrm{~V}$ and Vpass is $4.5,7,10 \mathrm{~V}$, respectively.

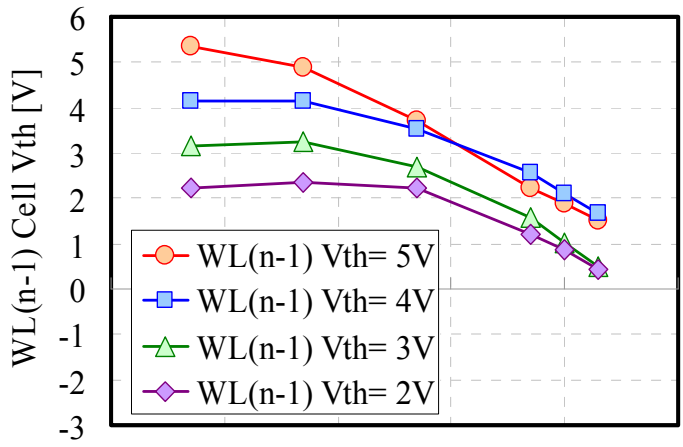

$1 \mathrm{E}-5 \quad 1 \mathrm{E}-4 \quad 1 \mathrm{E}-3 \quad 1 \mathrm{E}-2 \quad 1 \mathrm{E}-1 \quad 1 \mathrm{E}+0$

WL(n) Program Stress Time $[\mathrm{s}]$

Figure 6. The Vth's reduction of $\mathrm{WL}(\mathrm{n}-1)$ with the initial Vth. The program stress voltage is $26 \mathrm{~V}$ and the pass voltage is $4.5 \mathrm{~V}$. This result is the measured result for a single cell.

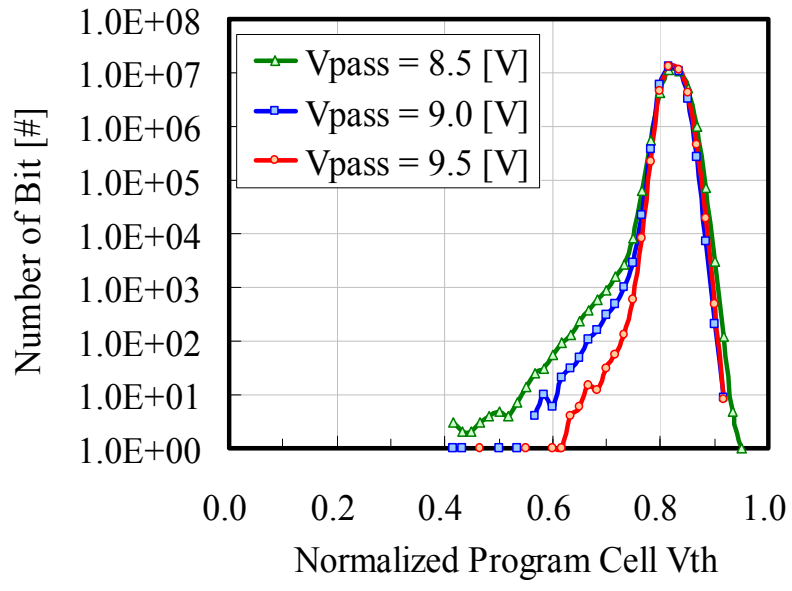

Figure 7. The distribution of program cell Vth after program of the main chip, where Vpass is $8.5,9.0,9.5 \mathrm{~V}$, respectively.
Until now, we have shown the results under the extreme program condition of the TEST modules. The program time of the main chip is shorter and Vpass in the main chip is higher than the program stress condition given for the TEST Module. So we cannot observe this phenomenon in the main cell more than Sub-40nm. Fig. 7 has shown the normalized Vth distribution after all word lines are programmed to high state(ex. 4.7V) from lower word line to higher word line at the main chip in order. The gate design rule of the main chip is sub-30nm. The under tail of Fig.7 is generated at the WL(n-1) when the WL(n) is programmed. The final word line without the upper word line no has the under tail of the Vth distribution. As the pass voltage is increasing, the tail of Vth distribution is decreasing. In the main cell, the dependence upon the program voltage and the initial Vth of WL(n-1) are the same tendency as the results in the test modules. Because the reduction of Vth in the test module have a very large distribution, as shown in Fig. 3 , the program interference phenomenon in the main cell is appeared as a tail.

\section{DISCUSSION}

It is important to know that the variation rate of the victim cell's Vth with the program stress voltage in Fig. 4 and with the pass voltage in Fig. 5 are different. The variation rate of the Vth's reduction for the program stress voltage is from 0.9 to 1.5 and the variation rate of the Vth's reduction for the pass volatge is from 0.65 to 0.8 . From these results, we first assumed that the decrement of the victim cell's Vth is caused by the leakage current due to the potential difference between the control gate of the program word line and the floating gate of the victim cell. The coupling ratio of the sub- $30 \mathrm{~nm}$ node used for this experiment is around 0.68 coming from 3D simulation. The result of Fig. 6 also supports that this program interference is caused by the potential difference between the control gate and the floating gate. Because the Vth of the victim cell in Fig.2 is decreasing after the one of the program stress cell is saturated, the potential difference between the floating gates is not the cause of the charge loss. Even if it is so that the program interference is due to the potential difference between the control gate and the floating gate, is it FN tunneling current? The Fig. 8 is a simulation result of the electric field at $\mathrm{t}=0$ for $30 \mathrm{~nm}$ node. The simulation was performed in 3D-structure with practical dimension reflecting doping on Si channel, poly-Si floating gates, and control gates.

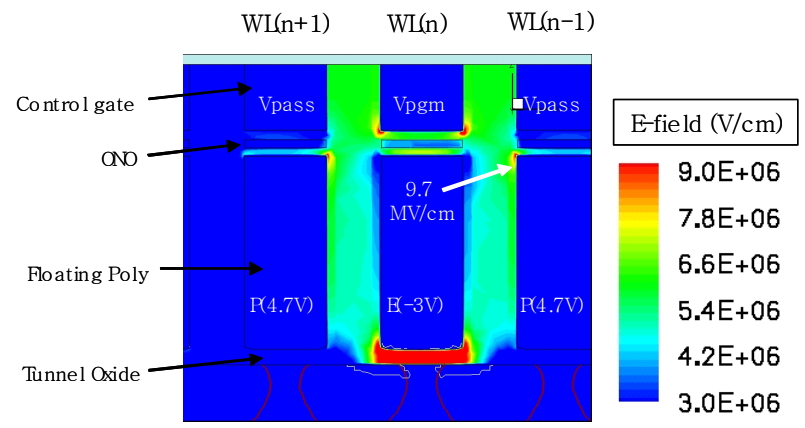

Figure 8. The simulation result of the electric field distribution under the program operation at $\mathrm{t}=0$, where the Vpgm is $24 \mathrm{~V}$ and the Vpass is $8.5 \mathrm{~V}$. 
The target Vth's of the floating gates are adjusted from Id$\mathrm{Vg}$ curve by controlling charges of the floating gates. The maximum electric field is formed between the top edge of the floating gate and the bottom edge of the control gate. We only have confirmed that the edge field is large enough to generate FN tunneling current through the simulation. Although more simulations have to be performed to prove the type of the leakage current, the measured results show that the Vth's reduction of the victim cell is similar one when the floating gate cell is erased by FN tunneling current. In the main cell, this program interference is decreased with the $\mathrm{P} / \mathrm{E}$ cycle as shown in Fig. 9. Because the program interference mentioned in this paper is caused by the leakage current between the floating gate and the control gate, the charge trapped in the tunnel oxide have nothing to do with it directly. The result in Fig. 9 is only due that the program voltage to adjust the same Vth is decreased as the $\mathrm{P} / \mathrm{E}$ cycle is progressed [8]. After 100 $\mathrm{P} / \mathrm{E}$ Cycle and $1000 \mathrm{P} / \mathrm{E}$ Cycle, the program speed is faster $0.3 \mathrm{~V}$ and $0.5 \mathrm{~V}$, respectively, than the initial program speed. Fig. 10 shows the $20^{\text {th }}$ measured results for the program interference at the same cell repeatedly.

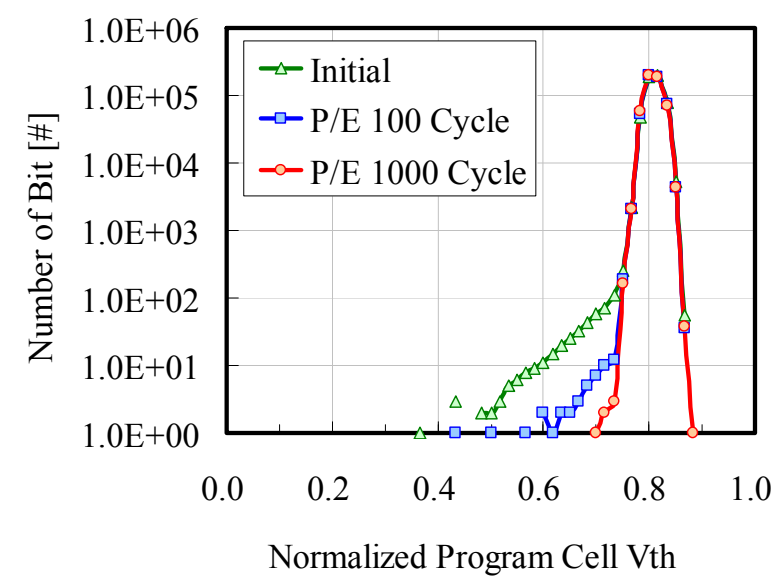

Figure 9. The distribution of program cell $\mathrm{Vth}$ after $\mathrm{P} / \mathrm{E}$ cycle at the main chip, where the program speed is faster $0.3 \mathrm{~V}$ and $0.5 \mathrm{~V}$, respectively, than the initial program speed after $100 \mathrm{P} / \mathrm{E}$ Cycles and $1000 \mathrm{P} / \mathrm{E}$ Cycles.

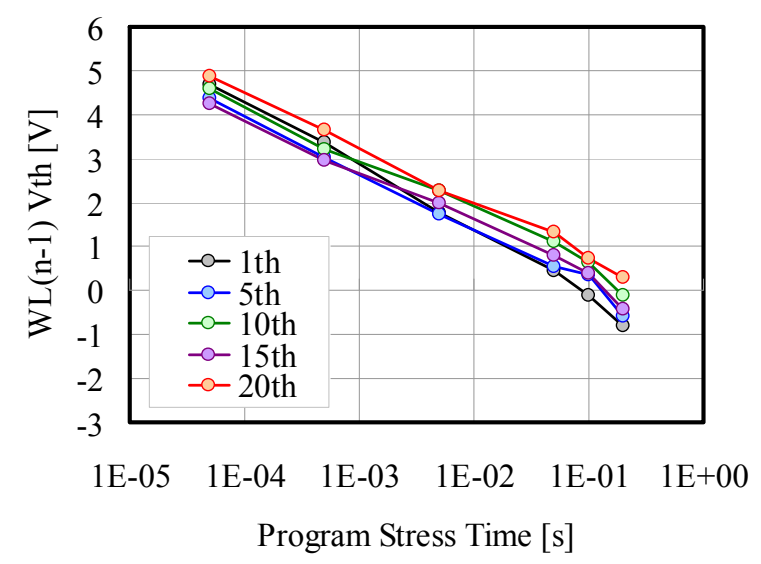

Figure 10. The 20th measured results for the program interference at the same cell in the test module repeatedly.
If the leakage current is related with the trap assisted tunneling, the measured results have to show any tendency. So we guess that this leakage current is supposed to be the FN like tunneling current.

\section{SCALING EFFECT}

We have measured these Vth's reductions in the NAND test modules with different gate design rules, which are from sub$30 \mathrm{~nm}$ to sub-50nm. Fig. 11 shows that the space between the adjacent WL gates is very critical to this phenomenon. The yaxis in Fig. 11 is the amount of the Vth reduction after $0.2 \mathrm{~s}$ of program stress. The each value of Fig. 11 is the mean of 20 points. Although the Vth's reductions are measured at different program voltage according to the gate design rule, all of the measured results are simply generalized with the electric field between the control gates. The normalized results with the electric field are given in Fig. 12, where the y-axis is the decrement of the victim cell's Vth after program stress for $0.2 \mathrm{~s}$.

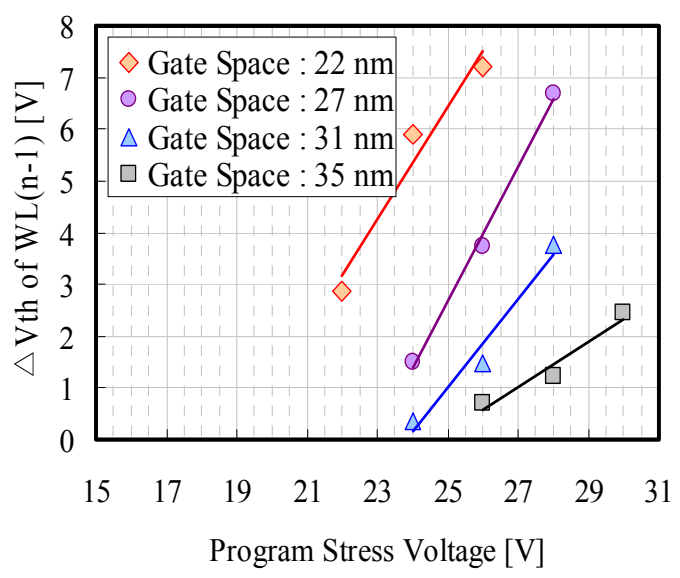

Figure 11. The Vth's reduction of WL(n-1) for samples with different gate space after $0.2 \mathrm{~s}$ of program stress, the pass voltage is $4.5 \mathrm{~V}$.

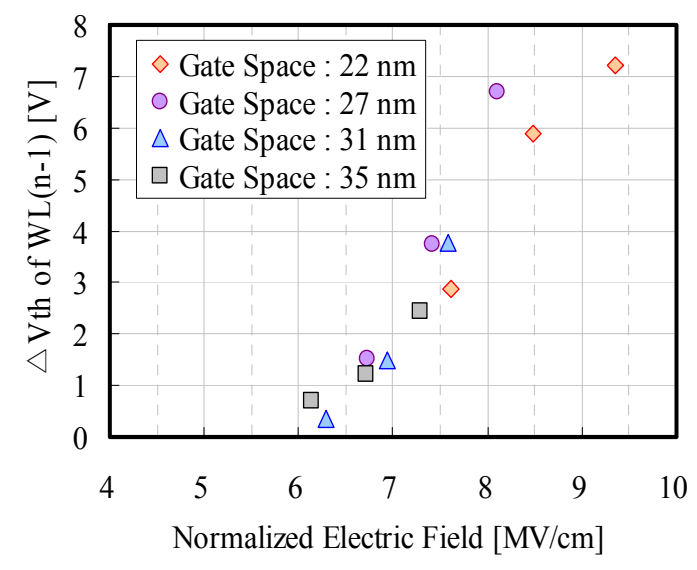

Figure 12. The Vth's reduction of WL(n-1) for samples with different gate space. The $\mathrm{x}$-axis is the electric field between the control gate of WL(n) and the WL(n-1). 
The reason for using the electric field between the control gates instead of the electric field between the WL(n)'s control gate (program stress cell) and the WL(n-1)'s floating gate (victim cell) is because it is difficult to use the representative value. As shown in Fig. 8, the electric field of the edge is susceptible to the shape and profile. If the coupling ratio of the floating gate cell is similar, the electric field between the control gates is a simple and proper parameter for representing this phenomenon in the industries. The Vth reduction of WL(n1) is measured above $6.0 \mathrm{MV} / \mathrm{cm}$ without the gate design rules. This means that the Vth reduction of $\mathrm{WL}(\mathrm{n}-1)$ is general phenomenon related to the electrical field between the floating gate and the adjacent control gate along the bit-line.

\section{CONCLUSION}

We have introduced a new scaling limitation of the floating gate cell in NAND Flash memory, which is the program interference. The charge loss of WL(n-1), when the WL(n) is programming, is attribute to the leakage current between WL(n)'s control gate and WL(n-1)'s floating gate. By extension, we have generalized this phenomenon with the electric field between the control gates. Because this program interference is rapidly increased as the space between the gates is decreased, the program speed and program operation condition should be determined with considering how this charge loss will be handled in the following nodes.

\section{REFERENCES}

[1] Chih-Yuan Lu, Tao-Cheng Lu, and Rich Liu, "Non-Volatile Memory Technology-Today and Tomorrow", in Proceedings of $13^{\text {th }}$ IPFA, 2006, pp. 18-23.

[2] T.Cho, Y. Lee, E. Kim, J.Lee, S. Choi, S. Lee, D. Kim, W. Han, Y. Lim, J. Lee, J. Choi, and K. Suh, "A dual-mode NAND flash memory: 1-Gb multilevel and high-performance 512-Mb single-level modes," SolidState Circuits, IEEE, 2004, pp. 895-900.

[3] K. Park, M. Kang, D. Kim, S. Hwang, B. Choi, Y. Lee, C. Kim, and K. Kim, "A zeroing cell-to-cell interference page architecture with temporary LSB storing and parallel MSB program scheme for MLC NAND flash memories," Solid-State Circuits, IEEE, 2008, pp. 919-928.

[4] Mincheol Park, Keonsoo Kim, Jong-Ho Park, and Jeong-Hyuck Choi, "Direct field effect of neighboring cell transistor on cell-to-cell interference of NAND flash cell arrays." IEEE Elrctron Deivce Letters, 2009, pp. 174-177.

[5] A. Nakamura, H. Moriya, T. Terano, H. Kosaka, A. Hashiguchi, K. Nomoto, I. Fujiwara, and T. Oda, "Narrow distribution of threshold voltage in 3-Mbit MONOS memory-cell array with F-N channel write and direct/F-N tunneling erase operation as a single transistor structure," IEEE Trans. Eectron Devices, 2004, pp. 895-900.

[6] Won-Seong Lee, "Future Memory Technologies", in Solid-State and Integrated-Circuit Technology, ICSICT $9^{\text {th }}$ international Conference, 2008, pp. 1-4.

[7] Kang-Deog Suh, Byung-Hoon Suh, Young-Ho Lim, et. al., “A 3.3V 32 $\mathrm{Mb}$ NAND flash memory with incremental step pulse programming scheme", Solid State Circuits, IEEE, 1995, pp. 1149-1156.

[8] T. Kamigaichi, F.Arai, H. Nitsuta, M. Endo, K. Nishihara, H. Takekida, et. al., "Floating Gate Super Multi Level NAND Flash Memory Technology for 30nm and Beyond", IEDM, IEEE, 2008, pp. 1-4. 